\title{
Gaps in communication skills of doctors
}

\section{Ariyananda PL}

Department of Internal Medicine, International Medical University, Malaysia.

\author{
Correspondence: Prof. Pilane Liyanage Ariyananda \\ e-mail: pariyananda@gmail.com \\ (1) https://orcid.org/0000-0002-1813-5419
}

\begin{abstract}
"I went to see a doctor for my backache, the doctor spoke only few words and prescribed few medicines to be collected from the pharmacy. I wanted to say more about my illness, but I did not get a chance as the doctor was in a hurry."
\end{abstract}

This is a familiar story. There are many things that patients would like to happen when they see a doctor. It is clear in the foregoing narration that this doctor-patient encounter fell far short of the expectations of the patient. Let us reflect why patients perceive that some consultations are far short of their expectations and whether such expectations are rational.

'Tomorrow's Doctors' published by the United Kingdom General Medical Council spells out competency level of communication skills required by a doctor as: "Doctors are expected to; communicate clearly, sensitively and effectively with patients, their relatives or other care givers, and colleagues from the medical and other professions, by listening, sharing and responding; communicate clearly, sensitively and effectively with individuals and groups regardless of their age, social, cultural or ethnic background" (1).

\section{What communication skills should a doctor possess?}

Spoken skills: Doctors are expected to possess generic communication skills such as speaking skills, writing skills, listening skills and non-verbal communication skills. Doctors are expected to use these skills during their interactions with patients and caregivers, and also when they interact with members of the healthcare team. During doctorpatient interactions, communications should be done with empathy and compassion. In addition to data gathering that takes place during history taking, three other important processes take place and they include rapport building, information sharing (including patient education) and counselling. It is known that hospital specialists give more emphasis to data gathering, whereas latter three aspects of history-taking are carried out better by general practitioners, psychologists, psychiatrists and palliative care physicians. During data gathering, more closed-ended questions are used, compared to open-ended questions that are used more frequently during information sharing and rapport building. During data gathering what one records is mainly biomedical data to find out about the disease (disease-centered approach) as opposed to inquiring about perspectives to find out about suffering (illness-centered approach) (2). As neither of these approaches alone are adequate, it is important for doctors to strike a balance between 'diseasecentered' approach and 'illness-centered' approach with a 'patient-centered' approach. 'Patient-centered model' is a hybrid of the 'disease-illness model' that was used earlier, which had two segments namely 'disease framework' or 'doctor's agenda' and 'illness framework' or 'patient's agenda'. The 'patient-centered' approach is more likely to address holistic care. Therefore, doctors should take a 'patient-centered' approach when engaging in history taking as it gives a balanced interaction (3). This requires fluency in the appropriate use of 'open ended and closed questions', attentive listening, and clarification of contents of the discussion as and when necessary, rephrasing questions, summarizing the conversations on and off as the consultation proceeds, and interpretation of verbal and non-verbal behavior. Such close interactions seem to develop a therapeutic relationship (4). It is important for doctors to learn 
and practice the art of' 'cue recognition', which may be expressed verbally and non-verbally. They should also learn how to respond to patients' emotions, be it negative or positive. Often emotions are expressed non-verbally. These skills are very useful in special situations like 'breaking bad news'. In the approach recommended by Neighbour in 1987, he mentions five aspects - connecting, summarizing, handing-over, safety-netting and housekeeping - that needs addressing (5). In addition to the approach mentioned above, other well-known methods of doctor-patient communications include: i) Calgary-Cambridge Model which has a structured questionnaire with about 70 questions that are clustered into subdivisions such as initiating the session, gathering information, building relationship, explanation about the illness and management plan, and closure (6); ii) 'Threefunction approach' with subdivisions including data gathering, rapport building and supporting patient's emotions, and counselling or education of patients regarding their illness (7); iii) 'E4 Model' which includes engaging (engage with the patient), empathizing (empathize with the patient), educating (educate the patient) and enlisting (involve the patient in decision making and adhering to treatment) (8), and iv) 'Four-habits Model' of Franken and Stein which includes investing in the beginning, eliciting the patient's perspective, demonstrating empathy and investing in the end (9).

There are things that doctors need to consciously avoid to prevent breakdown in communication. These include getting distracted by other activities, avoiding eye contact, having a blank stare, reading the computer screen / smart phone, or allowing frequent interruptions such as telephone calls and nurses' questions. Doctors should not write and listen at the same time. It can lead to loss of information, both verbally or non-verbally. When listening and questioning, doctors should look at the patient at the same eye level as the patient. Looking down at a patient from a higher point gives the impression of the doctor being dominant with minimal or no empathy and supressing a patient's attempts to carry out a conversation.

There are techniques which could facilitate clinical communication such as asking 'Is there anything else you would like to talk about? 'or sharing the doctor' own life experiences, which may help the patient's well-being. Patients always have questions to ask the doctor, but many doctors do not give them a chance as noted in the narration given at the outset. Doctors should always inquire from patients about their ideas, concerns and expectations. Educating the patient and any personal caregiver is important as it facilitates the management and improves the outcome. Therefore, doctors should make it a habit to tell the patient about the diagnosis, aetiology, treatment, prognosis and functional consequences (impact on life) as far as possible in lay language. Doctors should always verify whether the patient has understood what has been told, as understanding increases the compliance to treatment.

Additional spoken skills of doctors are required when carrying out telephone conversations. Seeking advice from superiors regarding patient management (including emergency care), arranging urgent investigations, arranging special aspects to treatment such as rehabilitation and arranging urgent patient transfers are some instances where doctors have to engage in telephone conversations. This is an important communication skill as telephone conversations involve delivering and receiving essential information in a clear, structured format, succinctly and courteously within a short time. The person delivering the information must ensure that the listener has fully understood what is being conveyed. The person receiving the information must listen attentively to the person at the other end of the telephone line, understand what is being conveyed and react appropriately and courteously. In case of doubt, clarifications should be sought. Doctors should seek advice from their superiors or from doctors in the appropriate specialty, as and when needed. Amongst doctors in Sri Lanka, there is plenty of room for improvement of these skills.

Written skills: Written communications are important for the safe practice of medicine. In Sri Lanka, doctors write down their clinical notes in English mainly in formats such as histories, follow-up notes, operation notes, diagnosis cards and referral letters. These documentations need to be precise but adequate (without unacceptable abbreviations), clear, accurate and timely. Often doctors in Sri Lanka fail to meet this requirement 
which is evident in consultation notes written by specialist doctors when engaging in private practice. This practice leads to bad role modelling. Similar poor quality writings are commonly seen in state hospitals as well.

Communication skills to handle special situations: There are several situations where doctors have to exercise special skills in communication to be effective such as when: i) interacting with patients with regards to risk and uncertainty, ii) responding to patients' emotions, iii) breaking bad news, iv) facilitating behaviour change through motivational interviewing, v) responding to medical errors and complaints, vi) handling mental health matters, vii) dealing with elderly patients, and viii) dealing with end of life issues. Doctors need special training to handle these situations effectively and sensitively, taking cultural and social contexts as well as role of the family into consideration.

\section{Concluding remarks}

Research has shown that when physicians consult a doctor for their own illnesses, they prefer their doctors to have at least the following two qualities. First, the physician providing care should be technically excellent; second, he or she should be empathic and humane (8). All doctors should treat their patients in the same way they themselves want to be treated.

\section{References}

1. General Medical Council, Outcomes for graduates (Tomorrow's Doctors), 2015. Retrieved from https://www.gmc-uk.org/-/media/documents/outcomesfor-graduates-jul-15-1216_pdf-61408029.pdf

2. Engel, G., The need for a new medical model: A challenge for biomedicine. Science, 1977; 196: 129-136.

3. Stewart M, Brown J, Boon H, Galajda J, Meredith L, Sangster M. Evidence on patient- doctor communication. Cancer Prevention and Control, 1999; 3: 25-30.

4. Ackerman SJ, Hilsenroth MJ, A review of therapist characteristics and techniques positively impacting the therapeutic alliance. Clinical Psychology Review, 2003; 23(1), 1-33.

5. Neighbour R. The inner consultation. $2^{\text {nd }}$ edn. Oxford: Radcliffe Publishing; 2004.

6. Kurtz SM, Silverman JD, Draper J Teaching and Learning Communication Skills in Medicine. Radcliffe Medical Press (Oxford); 1998.

7. Cohen Cole, S. The Medical Interview: The Three Function Approach. Mosby Year Book, St. Louis. 1991.

8. Keller VF \& Carroll JG, A new model for physician - patient communication. Patient Education and Counselling, 1994; 23: 131-140.

9. Frankel RM, Stein T, Getting the most out of the clinical encounter: the four habits model. J Med Pract Manage, 2001; 16: 184-91. 